\title{
Afrontamiento primario con elásticos vasculares en el manejo del cierre progresivo de fasciotomías*
}

\author{
Drs. ANDRÉS REYES D. ${ }^{1}$, STEPHANIE SIEGEL A. ${ }^{1}$, JOAQUÍN TORRES R. ${ }^{1}$, \\ CARLOS DEROSAS A. ${ }^{1}$, FRANCISCO ACUÑA D. ${ }^{1}$, LEOPOLDO MARINE M. ${ }^{2}$, \\ RODRIGO JULIO A. ${ }^{3}$, CRISTINA SAAVEDRA H ${ }^{1}$, RICARDO OBANDO V ${ }^{1}$., \\ CLAUDIA LEMUS R ${ }^{1}$., CLAUDIO SCHULTZ S. ${ }^{1}$
}

1 Equipo de Cirugía Vascular Hospital San José, Universidad de Santiago.

2 Equipo de Cirugía Vascular, Pontificia Universidad Católica.

3 Equipo de Cirugía Vascular Hospital del Salvador, Universidad de Chile. Santiago, Chile.

\section{Abstract}

\section{Vessel loops for primary closure of fasciotomies}

Background: Compartment syndrome of the extremity may occur after severe trauma with vascular lesions secondary to fractures, crushes or gunshots. To prevent it a fasciotomy must be done. Aim To report the use vessel loop shoelace technique for the progressive closure of the fasciotomy. Material and methods: Descriptive study of 24 patients aged $26 \pm 9$ years $(21$ males $)$ that required fasciotomy to prevent compartment syndromes. The fasciotomy wound was closed progressively using vessel loops anchored to the skin with staples or sutures, which were tightened progressively, according to the evolution. Results: The studied patients required a total of 56 fasciotomies. In all patients a complete or near complete closure of the wound was achieved. The mean closure time was $9.5 \pm 3.3$ days. Mean hospital stay was $12.3 \pm 4.3$ days. Conclusions: Vessel loop shoelace technique is effective for fasciotomy wound closure.

Key words: Vessel loop, fasciotomy, compartment syndrome.

\section{Resumen}

Frente al cada vez más frecuente manejo del trauma vascular en extremidades, debido a lesiones de distinta etiología, ya sea por accidentes de tránsito de alta velocidad, caídas de altura, o las crecientes tasas de lesiones en la vida civil por arma blanca y por arma de fuego, es que nos vemos enfrentados a múltiples tipos de lesiones vasculares que comprometen arterias y venas, asociados a extenso compromiso de partes blandas o a reintervenciones en períodos posteriores a las $6 \mathrm{hrs}$ post trauma. De este modo es que el manejo asociado de la fasciotomía como método de prevención o tratamiento del síndrome compartamental es fundamental. Se presentan un total de 24 casos en los que se efectuaron 56 fasciotomías primarias o secundarias como profilaxis o tratamiento del síndrome compartamental, en las que se utilizaron elásticos vasculares para el cierre progresivo del defecto cutáneo de las fasciotomías, para disminuir el tiempo de evolución y así evitar el uso de injertos de piel en el cierre de los defectos.

Palabras clave: Fasciotomías, trauma vascular, cierre de fasciotomías.

\footnotetext{
*Recibido el 7 de Enero de 2010 y aceptado para publicación el 17 de Marzo de 2010.

Correspondencia: Dr. Andrés Reyes D.

San José No 1196, Santiago, Chile. Fax: 02563832841

E-mail: reydusch@gmail.com
} 


\section{Introducción}

Ante el incremento de las lesiones vasculares provocadas por accidentes de tránsito, caídas de altura, y el creciente aumento de las agresiones por arma blanca y armas de fuego, es que nos vemos enfrentados a complejas lesiones vasculares de extremidades. Estas incluyen lesiones de vasos principales, arterias y venas, con extenso compromiso de partes blandas, fracturas y grandes hematomas compresivos; además asistimos a un número importante de reintervenciones por los equipos de cirugía vascular para efectuar reparaciones vasculares definitivas una vez que los equipos de emergencia han logrado el principal objetivo al recibir estos pacientes en las salas de trauma, controlando los daños para salvar la vida de los lesionados ${ }^{1}$.

De esta manera, se hace presente la indicación de realizar fasciotomías de protección, por lesiones combinadas de arterias y venas, lesiones de más de 6 horas de evolución desde el momento del trauma hasta su intervención, o con extenso compromiso de partes blandas, así como la realización de fasciotomías terapéuticas en pacientes que presentan síndrome compartamental en el contexto de la extensión del trauma ${ }^{2,3}$.

El objetivo de este trabajo, es dar a conocer los resultados en una serie de 24 pacientes que requirieron fasciotomías en el manejo inicial, con cierre progresivo con elásticos de las incisiones de piel de las fasciotomías, lo que disminuye de manera categórica el tiempo de hospitalización y la tasa de complicaciones asociadas a la fasciotomía, así como también la necesidad de coberturas posteriores de los defectos de piel mediante injertos dermoepidérmicos.

\section{Material y Método}

Estudio descriptivo y prospectivo de 24 casos de pacientes que presentaron lesiones vasculares o síndromes compartamentales en extremidades inferiores y superiores de distinta etiología, que requirieron fasciotomías de los compartimientos afectados, desde Octubre de 2007 hasta Junio de 2009. Se recogieron múltiples variables las que fueron analizadas en el programa SPSS 15.0.

\section{Técnica}

Todos los pacientes fueron intervenidos en pabellón central, en el contexto de pacientes graves con lesiones vasculares de distinto tipo o síndromes compartamentales en evolución.

La técnica descrita consiste en efectuar las distintas fasciotomías de manera habitual para liberar la presión de los compartimientos afectados. Seguido a esto, previo aseo y hemostasia de las zonas de apertura de piel y fascia, se procede a colocar múltiples corchetes para cierre de piel, o puntos simples de material no absorbible equidistantemente separados a modo de ojal, en los bordes superior e inferior de la piel abierta por la fasciotomía. Luego se entrelazan de forma cruzada tantos elásticos vasculares como sean necesarios para abarcar la totalidad de la longitud de los bordes de piel, anudando sus cabos libres, sin ejercer tensión sobre la piel. Se coloca posteriormente moltopren entre el músculo expuesto y elásticos a fin de poder manejar la herida como una lesión semicerrada, cubriendo luego con gasas sueltas y apósitos. Al segundo día y si la disminución de la tensión lo permite, se comienza a efectuar el ajuste progresivo de los elásticos vasculares, con técnicas estándar de asepsia y antisepsia, soltura de los nudos en los elásticos y reaproximación de los bordes de piel, manteniendo el concepto de afrontamiento sin tensión de los bordes para no caer en un posible síndrome compartamental posterior.

Esta maniobra se repite a diario y cuantas veces sean necesarias hasta obtener el cierre total del defecto de piel o la disminución de la brecha, de manera que pueda ser manejada en forma ambulatoria cuando el paciente se encuentre de alta de las lesiones primarias que determinaron la realización de las fasciotomías. Todas las heridas son cerradas y suturadas finalmente con material no reabsorbible a puntos separados o corchetes de piel $^{4}$ (Figuras de 1 a 6 ).

\section{Resultados}

Se describen un total de 24 casos, en quienes fue necesario realizar 56 fasciotomías, en los distintos compartimientos afectados, con una distribución de 21 hombres (87\%) y 3 mujeres (13\%) (Figura 7) y una edad promedio de $26 \pm 9$ años. El 54,17\% de las lesiones fueron exclusivamente arteriales, el $4,17 \%$ venosas y el $41,67 \%$ de ambos vasos simultáneamente (Tabla 1). En relación al mecanismo del traumatismo, las lesiones por arma de fuego se presentan como las más frecuentes correspondiendo al $50 \%$ de los casos siendo la extremidad inferior la más lesionada, datos que se resumen en la Tabla 2.

Todos los pacientes fueron egresados de pabellón a la Unidad de Paciente Crítico y posteriormente trasladados a sala de cirugía. En todos se comenzó a efectuar el ajuste progresivo y diario de los elásticos vasculares desde el día posterior a la intervención si las condiciones de tensión de la extremidad afectada así lo permitían.

En 3 casos, los pacientes presentaron lesiones 


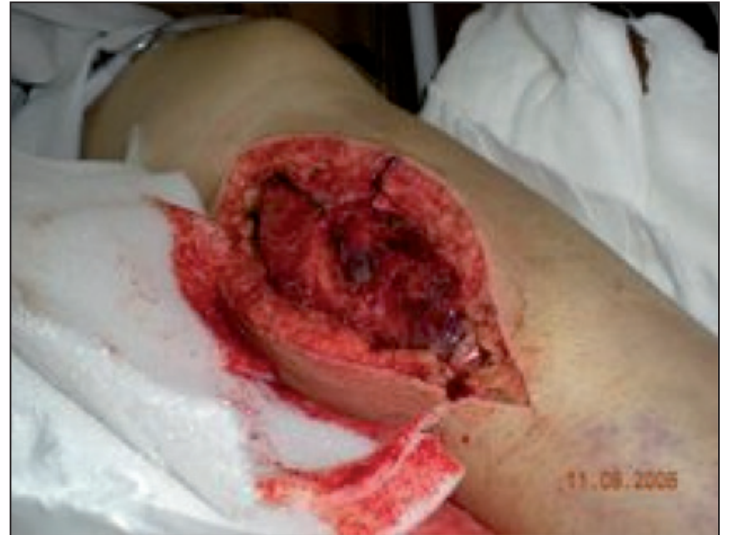

Figura 1. Fasciotomía.

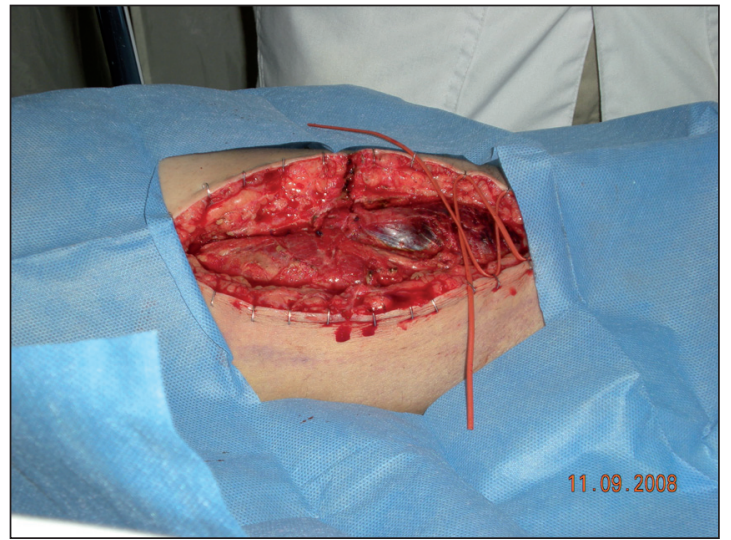

Figura 3. Instalación de elásticos.

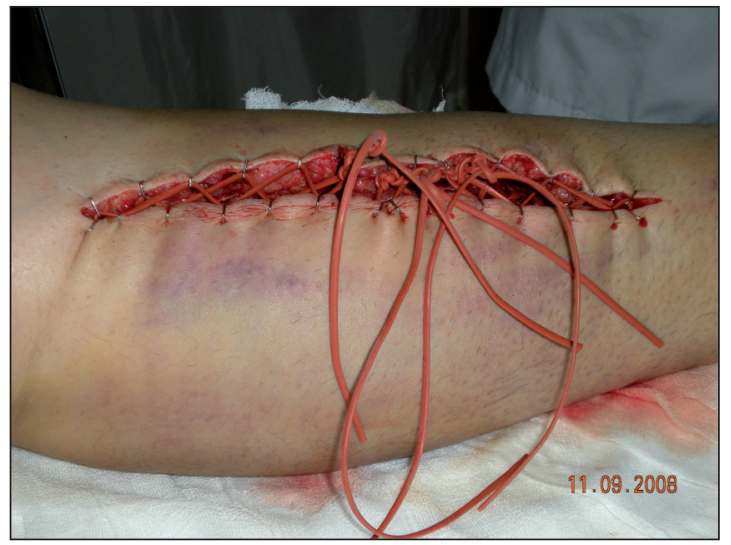

Figura 5. Cierre progresivo.

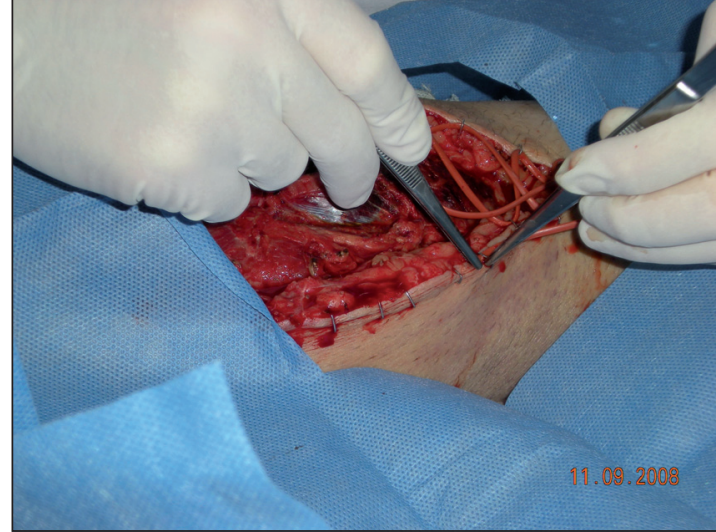

Figura 2. Instalación corchetes y elásticos.

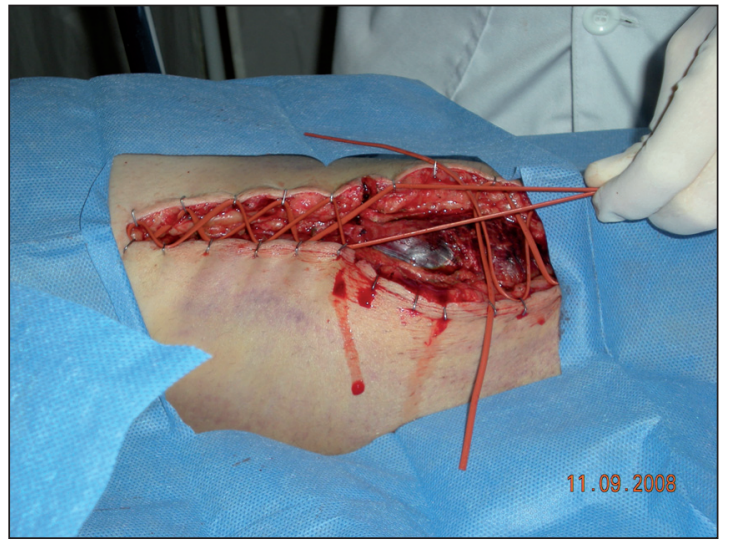

Figura 4. Ajuste de elásticos.

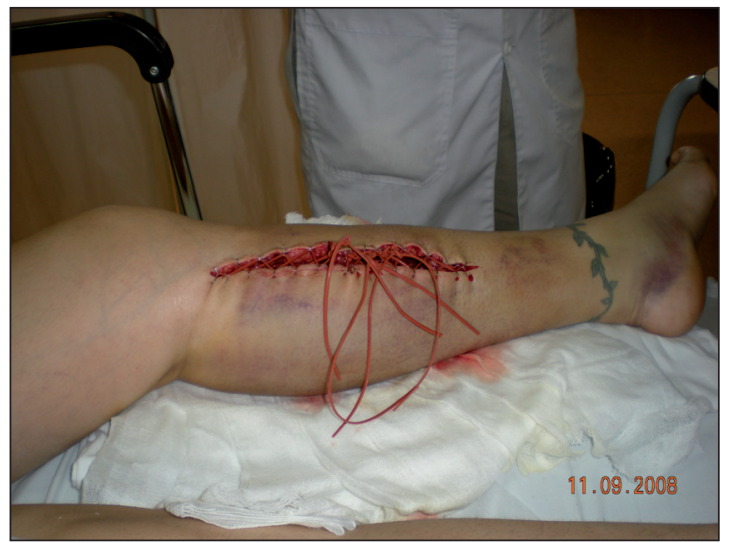

Figura 6. Elásticos previo a cierre definitivo. 


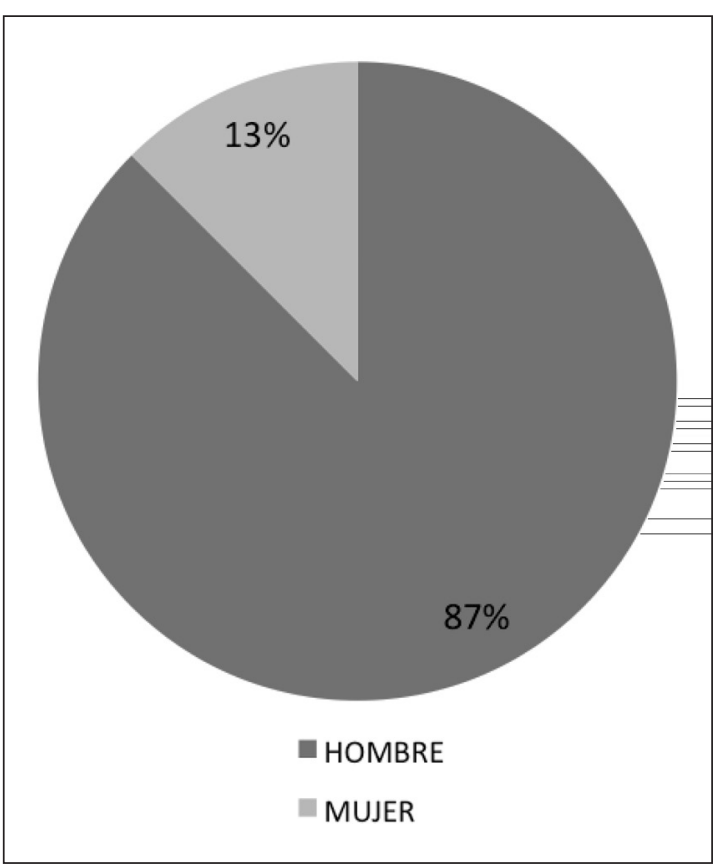

vasculares de extremidad superior. En uno de ellos se efectuó fasciotomía de ambos compartimientos del antebrazo, en los otros sólo del compartimiento anterior, con un total de 4 fasciotomías (Tabla 3).

En 7 casos, fue necesaria la realización de fasciotomías de 4 compartimientos en la extremidad inferior lesionada, con un total de 28 fasciotomías. En 11 pacientes, se requirió efectuar fasciotomías en 2 compartimientos de las piernas lesionadas, con un total de 22 fasciotomías. En 6 pacientes, se realizaron fasciotomías de sólo un compartimiento de la extremidad inferior afectada, con un total de 6 fasciotomías. De esta manera en un universo de 24 pacientes con diferentes diagnósticos de trauma vascular atendidos, se realizaron un total de 56 fasciotomías, (Figura 8) manejadas con cierre progresivo del defecto de piel con elásticos vasculares con distintas ubicaciones anatómicas. Dos pacientes requirieron injerto dermoepidérmico por defecto del cierre de una de sus fasciotomías mayor a $4 \mathrm{cms}$ de ancho.

En un caso de alta precoz por fuga del paciente, no fue necesario realizar injerto de piel, ya que al

Figura 7. Distribución de pacientes según sexo.

Tabla 1. Distribución de pacientes según vaso y ubicación anatómica

\begin{tabular}{lcccc}
\hline Vaso afectado & Arteria & Vena & Arteria + vena & Total \\
\hline Cubital & 1 & 0 & 0 & $1(4,2 \%)$ \\
Radial & 1 & 0 & 0 & $(4,2 \%)$ \\
Humeral & 1 & 0 & 1 & $2(8,3 \%)$ \\
Femoral común & 2 & 1 & 1 & $4(16,7 \%)$ \\
Femoral superficial & 4 & 0 & 3 & $7(29,2 \%)$ \\
Poplítea proximal & 1 & 0 & 2 & $3(12,5 \%)$ \\
Poplítea distal & 1 & 0 & 2 & $3(12,5 \%)$ \\
Tibial posterior & 2 & 0 & 1 & $3(12,5 \%)$ \\
Total & $13(54,2 \%)$ & $1(4,2 \%)$ & $10(41,6 \%)$ & $24(100 \%)$ \\
\hline
\end{tabular}

Los paréntesis corresponden al porcentaje total de la respectiva fila o columna.

Tabla 2. Distribución de pacientes según mecanismo del trauma y extremidad afectada

\begin{tabular}{lccc}
\hline Mecanismo & Extremidad superior & Extremidad inferior & Total \\
\hline Arma de fuego & 1 & 11 & $12(50 \%)$ \\
Arma blanca & 1 & 6 & $7(29,2 \%)$ \\
Aplastamiento & 1 & 1 & $2(8,3 \%)$ \\
Herramienta cortante & 1 & 0 & 1 \\
Accidente de tránsito & 0 & 2 & $2(4,2 \%)$ \\
Total & $4(16,7 \%)$ & $20(83,3 \%)$ & $24(100 \%)$ \\
\hline
\end{tabular}

Los paréntesis corresponden al porcentaje total de la respectiva fila o columna. 
Tabla 3. Distribución de pacientes según ubicación de la fasciotomía

\begin{tabular}{lcc}
\hline Ubicación fasciotomía & Frecuencia & $\mathbf{\%}$ \\
\hline Antebrazo anterior & 3 & 12,50 \\
Antebrazo anterior y posterior & 1 & 4,17 \\
Muslo & 5 & 20,83 \\
Muslo y pierna & 7 & 29,17 \\
Pierna & 5 & 20,83 \\
Pierna medial & 3 & 12,50 \\
Total & 24 & 100,00 \\
\hline
\end{tabular}

Tabla 4. Rangos y promedios en días \pm desviación standard (SD) de cierre de la fasciotomía y los días de hospitalización

\begin{tabular}{lccc}
\hline & n & $\begin{array}{c}\text { Extremos } \\
\text { en días }\end{array}$ & Promedio \pm SD \\
\hline Cierre & 24 & $5-16$ & $9,5 \pm 3,31$ \\
Hospitalización & 24 & $7-22$ & $12,38 \pm 4,33$ \\
\hline
\end{tabular}

momento del control (2 $1 / 2$ semanas luego de la fuga), la herida estaba limpia, granulada y cerrando por segunda intención.

La hospitalización promedio fue de 12,38 $\pm 4,33$ días, con un rango de 7 a 22 días y con un tiempo de cierre promedio de 9,5 $\pm 3,31$ días (Tabla 4).

\section{Discusión}

En todos los otros casos, se logró el cierre completo o casi total de los defectos de piel, no siendo necesarias nuevas intervenciones para cobertura o manejo de infecciones o sangrado de bordes, con un tiempo promedio de cierre de las fasciotomías de $9,5 \pm 3,31$ días.

Es así como concluimos que esta es una técnica sencilla, altamente efectiva en disminuir los tiempos de cierre de los defectos cutáneos de las fasciotomías (en comparación a lo publicado ${ }^{5-6}$ y a la experiencia en nuestro centro). Creemos que es reproducible en todo tipo de centros hospitalarios que cuenten con los insumos necesarios.

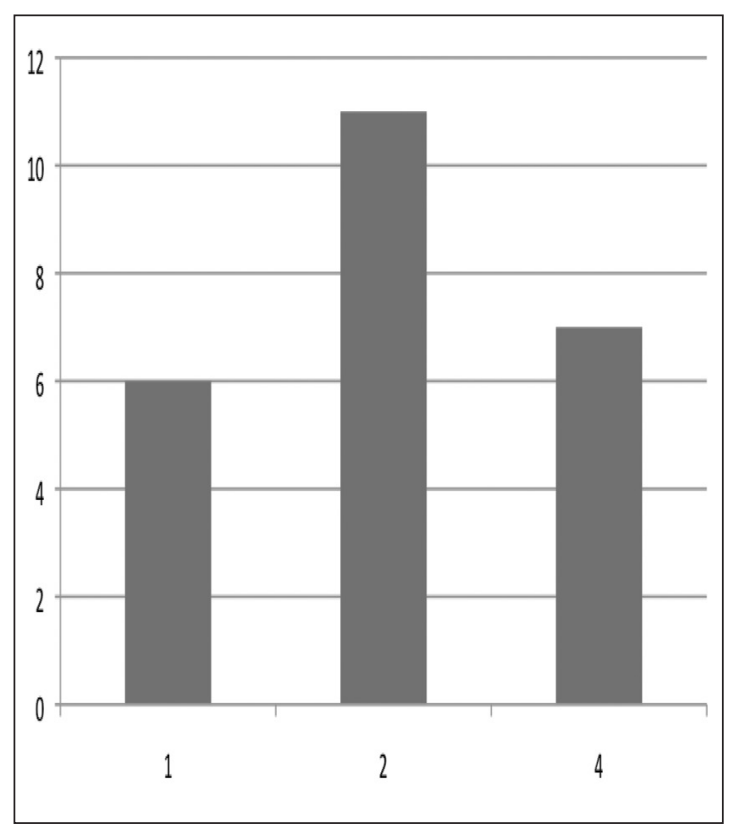

Figura 8. Distribución de pacientes según el número de fasciotomías realizadas.

\section{Referencias}

1. Bhattacharyya, Timothy, Vrahas, Mark S. The MedicalLegal Aspects of Compartment Syndrome. J Bone Joint Surg Am 2004; 86: 864-868.

2. Dente CJ, Feliciano DV, Rozycki GS, Cava RA, Ingram WL, Salomone JP, et al. A review of upper extremity fasciotomies in a level I Trauma Center. Am Surg 2004; 70: 1088-1093.

3. Taylor RC, Reitsma BJ, Sarazin S, Bell MG. Early results using a dynamic method for delayed primary closure of fasciotomy wounds. J Am Coll Surg 2003; 197: 872-878.

4. Mariné L. Técnica de cierre diferido de incisiones de fasciotomías con elásticos. Rev Chil Cir 2009; 61: 203205.

5. Barnea Y, Gur E, Amir A, Leshem D, Zaretski A, Miller E, et al. Delayed primary closure of fasciotomy wounds with wisebands, a skin-and soft tissue strecht device. Injury 2006; 37: 561-566.

6. Zorrilla P, Marín A, Gómez LA, Salido JA. Shoelace technique for gradual closure of fasciotomy wounds. J Trauma 2005; 59: 1515-1517. 